\title{
Classification of Ehrhart polynomials of integral simplices
}

\author{
Akihiro Higashitani $\|^{t}$ \\ Department of Pure and Applied Mathematics, Graduate School of Information Science and Technology, Osaka \\ University, Toyonaka, Osaka 560-0043, Japan
}

\begin{abstract}
Let $\delta(\mathcal{P})=\left(\delta_{0}, \delta_{1}, \ldots, \delta_{d}\right)$ be the $\delta$-vector of an integral convex polytope $\mathcal{P}$ of dimension $d$. First, by using two well-known inequalities on $\delta$-vectors, we classify the possible $\delta$-vectors with $\sum_{i=0}^{d} \delta_{i} \leq 3$. Moreover, by means of Hermite normal forms of square matrices, we also classify the possible $\delta$-vectors with $\sum_{i=0}^{d} \delta_{i}=4$. In addition, for $\sum_{i=0}^{d} \delta_{i} \geq 5$, we characterize the $\delta$-vectors of integral simplices when $\sum_{i=0}^{d} \delta_{i}$ is prime.

Résumé. Soit $\delta(\mathcal{P})=\left(\delta_{0}, \delta_{1}, \ldots, \delta_{d}\right)$ le $\delta$-vecteur d'un polytope intégrante de dimension $d$. Tout d'abord, en utilisant deux bien connus des inégalités sur $\delta$-vecteurs, nous classons les $\delta$-vecteurs possibles avec $\sum_{i=0}^{d} \delta_{i} \leq 3$. En outre, par le biais de Hermite formes normales, nous avons également classer les $\delta$-vecteurs avec $\sum_{i=0}^{d} \delta_{i}=4$. De plus, pour $\sum_{i=0}^{d} \delta_{i} \geq 5$, nous caractérisons les $\delta$-vecteurs des simplex inégalités lorsque $\sum_{i=0}^{d} \delta_{i}$ est premier.
\end{abstract}

Keywords: Ehrhart polynomial, $\delta$-vector, integral convex polytope, integral simplex.

\section{Introduction}

One of the most attractive problems on enumerative combinatorics of convex polytopes is to find a combinatorial characterization of the Ehrhart polynomials of integral convex polytopes. In particular, the $\delta$-vectors of integral simplices play an important and interesting role.

Let $\mathcal{P} \subset \mathbb{R}^{N}$ be an integral polytope, i.e., a convex polytope any of whose vertices has integer coordinates, of dimension $d$, and let $\partial \mathcal{P}$ denote the boundary of $\mathcal{P}$. Given a positive integer $n$, we define

$$
i(\mathcal{P}, n)=\left|n \mathcal{P} \cap \mathbb{Z}^{N}\right| \text { and } i^{*}(\mathcal{P}, n)=\left|n(\mathcal{P} \backslash \partial \mathcal{P}) \cap \mathbb{Z}^{N}\right|,
$$

where $n \mathcal{P}=\{n \alpha: \alpha \in \mathcal{P}\}, n(\mathcal{P} \backslash \partial \mathcal{P})=\{n \alpha: \alpha \in \mathcal{P} \backslash \partial \mathcal{P}\}$ and $|X|$ is the cardinality of a finite set $X$. The systematic study of $i(\mathcal{P}, n)$ originated in the work of Ehrhart [2], who established the following fundamental properties:

(0.1) $i(\mathcal{P}, n)$ is a polynomial in $n$ of degree $d$;

$(0.2) i(\mathcal{P}, 0)=1$;

\footnotetext{
${ }^{\dagger}$ The author is supported by JSPS Research Fellowship for Young Scientists.

1365-8050 @ 2012 Discrete Mathematics and Theoretical Computer Science (DMTCS), Nancy, France
} 
(0.3) (loi de réciprocité) $i^{*}(\mathcal{P}, n)=(-1)^{d} i(\mathcal{P},-n)$ for every integer $n>0$.

We say that $i(\mathcal{P}, n)$ is the Ehrhart polynomial of $\mathcal{P}$. We refer the reader to [1, Chapter 3], [3, Part II] or [10. pp. 235-241] for the introduction to the theory of Ehrhart polynomials.

We define the sequence $\delta_{0}, \delta_{1}, \delta_{2}, \ldots$ of integers by the formula

$$
(1-\lambda)^{d+1} \sum_{n=0}^{\infty} i(\mathcal{P}, n) \lambda^{n}=\sum_{i=0}^{\infty} \delta_{i} \lambda^{i}
$$

Then the basic facts $(0.1)$ and $(0.2)$ on $i(\mathcal{P}, n)$ together with a fundamental result on generating function [10, Corollary 4.3.1] guarantee that $\delta_{i}=0$ for every $i>d$. We say that the sequence (resp. the polynomial)

$$
\delta(\mathcal{P})=\left(\delta_{0}, \delta_{1}, \ldots, \delta_{d}\right) \quad\left(\text { resp. } \delta_{\mathcal{P}}(t)=\sum_{i=0}^{d} \delta_{i} t^{i}\right)
$$

which appears in 1 is called the $\delta$-vector (resp. the $\delta$-polynomial) of $\mathcal{P}$. Alternate names of $\delta$-vectors are, for example, Ehrhart h-vector, Ehrhart $\delta$-vector or $h^{*}$-vector. By the reciprocity law (0.3), one has

$$
\sum_{n=1}^{\infty} i^{*}(\mathcal{P}, n) \lambda^{n}=\frac{\sum_{i=0}^{d} \delta_{d-i} \lambda^{i+1}}{(1-\lambda)^{d+1}}
$$

The following properties on $\delta$-vectors are well known:

- By 1 , one has $\delta_{0}=i(\mathcal{P}, 0)=1$ and $\delta_{1}=i(\mathcal{P}, 1)-(d+1)=\left|\mathcal{P} \cap \mathbb{Z}^{N}\right|-(d+1)$.

- By 2 one has $\delta_{d}=i^{*}(\mathcal{P}, 1)=\left|(\mathcal{P} \backslash \partial \mathcal{P}) \cap \mathbb{Z}^{N}\right|$. In particular, we have $\delta_{1} \geq \delta_{d}$.

- Each $\delta_{i}$ is nonnegative [9].

- If $\delta_{d} \neq 0$, then one has $\delta_{1} \leq \delta_{i}$ for every $1 \leq i \leq d-1$ [4].

- It follows from 2 that

$$
\max \left\{j: \delta_{j} \neq 0\right\}+\min \left\{k: k(\mathcal{P}-\partial \mathcal{P}) \cap \mathbb{Z}^{N} \neq \emptyset\right\}=d+1 .
$$

- When $d=N$, the leading coefficient of $i(\mathcal{P}, n)$, which coincides with $\sum_{i=0}^{d} \delta_{i} / d$ !, is equal to the usual volume of $\mathcal{P}$ [10, Proposition 4.6.30]. In general, the positive integer $\sum_{i=0}^{d} \delta_{i}$ is called the normalized volume of $\mathcal{P}$.

On the classification problem of the $\delta$-vectors of integral convex polytopes, when we consider the possible $\delta$-vectors of small dimensions, all of them are essentially given in [8] when $d=2$. However, the possible $\delta$-vectors are presumably open when $d \geq 3$.

In this article, we discuss the $\delta$-vectors of small normalized volumes. In particular, the $\delta$-vectors of integral simplices play a crucial role when $\sum_{i=0}^{d} \delta_{i} \leq 4$.

A brief overview of this article is as follows. After reviewing some well-known technique how to compute the $\delta$-vectors of integral simplices in Section 1, we study the possible $\delta$-vectors of $\sum_{i=0}^{d} \delta_{i} \leq 3$ 
by using two well-known inequalities on $\delta$-vectors in Section 2. In Section 3, we consider the case where $\sum_{i=0}^{d} \delta_{i}=4$ by considering all the $\delta$-vectors of all the integral simplices up to some equivalence. In Section 4 , we discuss the $\delta$-vectors of integral simplices when $\sum_{i=0}^{d} \delta_{i}$ is prime and we classify the possible $\delta$-vectors of integral simplices of $\sum_{i=0}^{d} \delta_{i}=5$ and 7 .

\section{Review on the computation of the $\delta$-vectors of integral simplices}

Before proving our theorems, we recall a combinatorial technique to compute the $\delta$-vector of an integral simplex.

Given an integral simplex $\mathcal{F} \subset \mathbb{R}^{N}$ of dimension $d$ with the vertices $v_{0}, v_{1}, \ldots, v_{d}$, we set

$$
\begin{aligned}
& S=\left\{\sum_{i=0}^{d} r_{i}\left(v_{i}, 1\right) \in \mathbb{R}^{N+1}: 0 \leq r_{i}<1\right\} \cap \mathbb{Z}^{N+1} \text { and } \\
& S^{*}=\left\{\sum_{i=0}^{d} r_{i}\left(v_{i}, 1\right) \in \mathbb{R}^{N+1}: 0<r_{i} \leq 1\right\} \cap \mathbb{Z}^{N+1} .
\end{aligned}
$$

We define the degree of an integer point $(\alpha, n) \in S\left((\alpha, n) \in S^{*}\right)$ with $\operatorname{deg}(\alpha, n)=n$, where $\alpha \in \mathbb{Z}^{N}$ and $n \in \mathbb{Z}_{\geq 0}$. Let $\delta_{i}=|\{\alpha \in S: \operatorname{deg} \alpha=i\}|$ and $\delta_{i}^{*}=\left|\left\{\alpha \in S^{*}: \operatorname{deg} \alpha=i\right\}\right|$. Then we have

Lemma 2.1 Work with the same notation as above. Then we have

(a)

$$
\sum_{n=0}^{\infty} i(\mathcal{F}, n) \lambda=\frac{\delta_{0}+\delta_{1} \lambda+\cdots+\delta_{d} \lambda^{d}}{(1-\lambda)^{d+1}}
$$

(b)

$$
\sum_{n=0}^{\infty} i\left(\mathcal{F}^{*}, n\right) \lambda=\frac{\delta_{1}^{*} \lambda+\cdots+\delta_{d+1}^{*} \lambda^{d+1}}{(1-\lambda)^{d+1}}
$$

(c)

$$
\delta_{i}^{*}=\delta_{d+1-i} \text { for } 1 \leq i \leq d+1 .
$$

We also recall the following

Lemma 2.2 [1, Theorem 2.4] Suppose that $\left(\delta_{0}, \delta_{1}, \ldots, \delta_{d}\right)$ is the $\delta$-vector of an integral convex polytope of dimension $d$. Then there exists an integral convex polytope of dimension $d+1$ whose $\delta$-vector is $\left(\delta_{0}, \delta_{1}, \ldots, \delta_{d}, 0\right)$.

Note that the required $\delta$-vector is obtained by forming the pyramid over the integral convex polytope. 


\section{Two well-known inequalities on $\delta$-vectors}

In this section, we present two well-known inequalities on $\delta$-vectors. By using them, we give the complete classification of the possible $\delta$-vectors of integral convex polytopes with $\sum_{i=0}^{d} \delta_{i} \leq 3$.

Let $s=\max \left\{i: \delta_{i} \neq 0\right\}$. Stanley [11] shows the inequalities

$$
\delta_{0}+\delta_{1}+\cdots+\delta_{i} \leq \delta_{s}+\delta_{s-1}+\cdots+\delta_{s-i}, \quad 0 \leq i \leq[s / 2]
$$

by using the theory of Cohen-Macaulay rings. On the other hand, the inequalities

$$
\delta_{d}+\delta_{d-1}+\cdots+\delta_{d-i} \leq \delta_{1}+\delta_{2}+\cdots+\delta_{i+1}, \quad 0 \leq i \leq[(d-1) / 2]
$$

appear in [4, Remark (1.4)]. A proof of the inequalities 4 is given by using combinatorics on convex polytopes.

Somewhat surprisingly, when $\sum_{i=0}^{d} \delta_{i} \leq 3$, the above inequalities 3 together with 4 give a characterization of the possible $\delta$-vectors. In fact,

Theorem 3.1 Given a finite sequence $\left(\delta_{0}, \delta_{1}, \ldots, \delta_{d}\right)$ of nonnegative integers, where $\delta_{0}=1$, which satisfies $\sum_{i=0}^{d} \delta_{i} \leq 3$, there exists an integral convex polytope $\mathcal{P} \subset \mathbb{R}^{d}$ of dimension d whose $\delta$-vector coincides with $\left(\delta_{0}, \delta_{1}, \ldots, \delta_{d}\right)$ if and only if $\left(\delta_{0}, \delta_{1}, \ldots, \delta_{d}\right)$ satisfies all inequalities 3 and 4 Moreover, all integral convex polytopes can be chosen to be simplices.

Note that the "Only if" part of Theorem 3.1 is obvious. Thus we may show the "If" part. Moreover, when $\sum_{i=0}^{d} \delta_{i}=1$, it is obvious that the possible sequence is only $(1,0, \ldots, 0)$ and this is a $\delta$-vector of some integral convex polytope, in particular, integral simplex. A sketch of a proof of the "If" part with $\sum_{i=0}^{d} \delta_{i}=2$ or 3 is as follows:

- When $\sum_{i=0} \delta_{i}=2$, the possible integer sequence looks like $(1,0, \ldots, 0, \underbrace{1}_{i}, 0, \ldots, 0) \in \mathbb{Z}^{d+1}$, where $\underbrace{1}_{i}$ means that $\delta_{i}=1$. On the other hand, we have $i \leq\lfloor(d+1) / 2\rfloor$ by 4 . Hence we may find an integral convex polytope, in particular, an integral simplex, whose $\delta$-vector coincides with that. Note that we may construct such simplex with $i=\lfloor(d+1) / 2\rfloor$ by virtue of Lemma2.2.

- When $\sum_{i=0} \delta_{i}=3$, we have two candidates of the possible integer sequences.

- When $(1,0, \ldots, 0, \underbrace{2}_{i}, 0, \ldots, 0) \in \mathbb{Z}^{d+1}$, similar discussions to the previous case can be applied.

- When $(1,0, \ldots, 0, \underbrace{1}_{i}, 0, \ldots, 0, \underbrace{1}_{j}, 0, \ldots, 0) \in \mathbb{Z}^{d+1}$, from 3 and 4 , we have the inequalities

$$
1 \leq i<j \leq d, \quad 2 i \leq j \text { and } i+j \leq d+1 .
$$

Once we can find an integral simplex whose $\delta$-vector coincides with that with $2 i=j$ and $i+j=d+1$, we can also construct an integral simplex whose $\delta$-vector is that for any integers $i$ and $j$ with the above inequalities. 
On the other hand, the following example shows that Theorem 3.1 is no longer true for the case where $\sum_{i=0}^{d} \delta_{i}=4$.

Example 3.2 The integer sequence $(1,0,1,0,1,1,0,0)$ cannot be the $\delta$-vector of any integral convex polytope of dimension 7 , although this satisfies the inequalities 3 and 4 . In fact, suppose, on the contrary, that there exists an integral convex polytope $\mathcal{P} \subset \mathbb{R}^{N}$ of dimension 7 with $\left(\delta_{0}, \delta_{1}, \ldots, \delta_{7}\right)=$ $(1,0,1,0,1,1,0,0)$ its $\delta$-vector. Since $\delta_{1}=0$, we know that $\mathcal{P}$ is a simplex. Let $v_{0}, v_{1}, \ldots, v_{7}$ be the vertices of $\mathcal{P}$. By using Lemma 2.1. one has $S=\{(0, \ldots, 0),(\alpha, 2),(\beta, 4),(\gamma, 5)\}$ and $S^{*}=$ $\left\{\left(\alpha^{\prime}, 3\right),\left(\beta^{\prime}, 4\right),\left(\gamma^{\prime}, 6\right),\left(\sum_{i=0}^{7} v_{i}, 7\right)\right\}$. Write $\alpha^{\prime}=\sum_{i=0}^{7} r_{i} v_{i}$ with each $0<r_{i} \leq 1$. Since $\left(\alpha^{\prime}, 3\right) \notin S$, there is $0 \leq j \leq 7$ with $r_{j}=1$. If there are $0 \leq k<\ell \leq 7$ with $r_{k}=r_{\ell}=1$, say, $r_{0}=r_{1}=1$, then $0<r_{q}<1$ for each $2 \leq q \leq 7$ and $\sum_{i=2}^{7} r_{i}=1$. Hence $\left(\alpha^{\prime}-v_{0}-v_{1}, 1\right) \in S$, a contradiction. Thus there is a unique $0 \leq j \leq 7$ with $r_{j}=1$, say, $r_{0}=1$. Then $\alpha=\sum_{i=1}^{7} r_{i} v_{i}$ and $\gamma=\sum_{i=1}^{7}\left(1-r_{i}\right) v_{i}$. Let $\mathcal{F}$ denote the facet of $\mathcal{P}$ whose vertices are $v_{1}, v_{2}, \ldots, v_{7}$ with $\delta(\mathcal{F})=\left(\delta_{0}^{\prime}, \delta_{1}^{\prime}, \ldots, \delta_{6}^{\prime}\right) \in \mathbb{Z}^{7}$. Then $\delta_{2}^{\prime}=\delta_{5}^{\prime}=1$. Since $\delta_{i}^{\prime} \leq \delta_{i}$ for each $0 \leq i \leq 6$, it follows that $\delta(\mathcal{F})=(1,0,1,0,0,1,0)$. This contradicts the inequalities 3 .

\section{Hermite normal forms with a given $\delta$-vector}

In this section, we give the complete classification of the possible $\delta$-vectors with $\sum_{i=0}^{d} \delta_{i}=4$ by means of Hermite normal forms. Moreover, it turns out that all the possible $\delta$-vectors with $\sum_{i=0}^{d} \delta_{i}=4$ can be chosen to be integral simplices.

Let $\mathbb{Z}^{d \times d}$ denote the set of $d \times d$ integer matrices. Recall that a matrix $A \in \mathbb{Z}^{d \times d}$ is unimodular if $\operatorname{det}(A)= \pm 1$. Given integral convex polytopes $\mathcal{P}$ and $\mathcal{Q}$ in $\mathbb{R}^{d}$ of dimension $d$, we say that $\mathcal{P}$ and $\mathcal{Q}$ are unimodularly equivalent if there exists a unimodular matrix $U \in \mathbb{Z}^{d \times d}$ and an integral vector $w \in \mathbb{Z}^{d}$, such that $\mathcal{Q}=f_{U}(\mathcal{P})+w$, where $f_{U}$ is the linear transformation in $\mathbb{R}^{d}$ defined by $U$, i.e., $f_{U}(\mathbf{v})=\mathbf{v} U$ for all $\mathbf{v} \in \mathbb{R}^{d}$. Clearly, if $\mathcal{P}$ and $\mathcal{Q}$ are unimodularly equivalent, then $\delta(\mathcal{P})=\delta(\mathcal{Q})$. Conversely, given a vector $v \in \mathbb{Z}_{>0}^{d+1}$, it is natural to ask what are all the integral convex polytopes $\mathcal{P}$ under unimodular equivalence, such that $\delta(\mathcal{P})=v$. We focus on this problem for simplices with one vertex at the origin. In addition, we do not allow any shifts in the equivalence, i.e., integral convex polytopes $\mathcal{P}$ and $\mathcal{Q}$ of dimension $d$ are equivalent if there exists a unimodular matrix $U$, such that $\mathcal{Q}=f_{U}(\mathcal{P})$.

For discussing the representative under this equivalence of the integral simplices with one vertex at the origin, we consider Hermite normal forms of square matrices.

Let $\mathcal{P}$ be an integral simplex in $\mathbb{R}^{d}$ of dimension $d$ with the vertices $v_{0}, v_{1}, \ldots, v_{d}$, where $v_{0}=$ $(0, \ldots, 0)$. Define $M(\mathcal{P}) \in \mathbb{Z}^{d \times d}$ to be the matrix with the row vectors $v_{1}, \ldots, v_{d}$. Then we have the following connection between the matrix $M(\mathcal{P})$ and the $\delta$-vector of $\mathcal{P}:|\operatorname{det}(M(\mathcal{P}))|=\sum_{i=0}^{d} \delta_{i}$. In this setting, $\mathcal{P}$ and $\mathcal{P}^{\prime}$ are equivalent if and only if $M(\mathcal{P})$ and $M\left(\mathcal{P}^{\prime}\right)$ have the same Hermite normal form, where the Hermite normal form of a nonsingular integral square matrix $B$ is a unique nonnegative lower triangular matrix $A=\left(a_{i j}\right) \in \mathbb{Z}_{>0}^{d \times d}$ such that $A=B U$ for some unimodular matrix $U \in \mathbb{Z}^{d \times d}$ and $0 \leq a_{i j}<a_{i i}$ for all $1 \leq j<i$. (See, e.g., [7, Chapter 4].) In other words, we can pick the Hermite normal form as the representative in each equivalence class. By considering the $\delta$-vectors of all the integral simplices arising from the Hermite normal forms $M$ with $\operatorname{det}(M)=4$, we obtain the following 
Theorem 4.1 Let $1+t^{i_{1}}+t^{i_{2}}+t^{i_{3}}$ be a polynomial in $t$ with $1 \leq i_{1} \leq i_{2} \leq i_{3} \leq d$. Then there exists an integral convex polytope $\mathcal{P} \subset \mathbb{R}^{d}$ of dimension $d$ whose $\delta$-polynomial coincides with $1+t^{i_{1}}+t^{i_{2}}+t^{i_{3}}$ if and only if $\left(i_{1}, i_{2}, i_{3}\right)$ satisfies

$$
i_{3} \leq i_{1}+i_{2}, i_{1}+i_{3} \leq d+1, i_{2} \leq\lfloor(d+1) / 2\rfloor
$$

and an additional condition

$$
2 i_{2} \leq i_{1}+i_{3} \text { or } i_{2}+i_{3} \leq d+1 .
$$

Moreover, all integral convex polytopes can be chosen to be simplices.

Note that the inequalities 5 follow from the inequalities 3 and 4 , that is to say, the condition 5 is automatically a necessary condition. Thus, the condition 6 is the new necessary condition on $\delta$-vectors when $\sum_{i=0}^{d} \delta_{i}=4$.

A sketct of a proof of this theorem is as follows:

- On the "If" part, we may construct integral simplices whose $\delta$-polynomials look like $1+t^{i_{1}}+t^{i_{2}}+t^{i_{3}}$ satisfying 5 and 6 . By characterizing the possible $\delta$-vectors of all the integral simplices arising from the Hermite normal forms $M$ with $\operatorname{det}(M)=4$, we can find such integral simplices.

- On the "Only if" part, we may show that if a polynomial $1+t^{i_{1}}+t^{i_{2}}+t^{i_{3}}$ satisfies 5 but does not satisfy 6, then $i_{1}>1$, i.e., an integral convex polytope with this $\delta$-polynomial is always a simplex. By characterizing the possible $\delta$-vectors of all the integral simplices arising from the Hermite normal forms $M$ with $\operatorname{det}(M)=4$, we can say that there exists no integral simplex whose $\delta$-polynomial is equal to $1+t^{i_{1}}+t^{i_{2}}+t^{i_{3}}$ not satisfying 6

Example 4.2 As we see in Example 3.2 the integer sequence $(1,0,1,0,1,1,0,0)$ cannot be the $\delta$-vector of any integral convex polytope of dimension 7 . In fact, since $8=2 i_{2}>i_{1}+i_{3}=7$ and $9=i_{2}+i_{3}>8$, there exists no integral convex polytope of dimension 7 whose $\delta$-polynomial is $1+t^{2}+t^{4}+t^{5}$. On the other hand, there exists an integral convex polytope of dimension 8 whose $\delta$-vector is $(1,0,1,0,1,1,0,0,0)$ since $9=i_{2}+i_{3}=d+1$.

Remark 4.3 We see that all the possible $\delta$-vectors can be obtained by integral simplices when $\sum_{i=0}^{d} \delta_{i} \leq$ 4 . However, the $\delta$-vector $(1,3,1)$ cannot be obtained from any integral simplex, while this is a possible $\delta$-vector of some integral convex polytope of dimension 2 . In fact, suppose that $(1,3,1)$ can be obtained from a simplex. Since $\min \left\{i: \delta_{i} \neq 0, i>0\right\}=1$ and $\max \left\{i: \delta_{i} \neq 0\right\}=2$, one has $\min \left\{i: \delta_{i} \neq 0, i>\right.$ $0\}=3-\max \left\{i: \delta_{i} \neq 0\right\}$, which implies that the assumption of [5, Theorem 2.3] is satisfied. Thus the $\delta$-vector must be shifted symmetric, a contradiction.

\section{Ehrhart polynomials of integral simplices with prime volumes}

From the previous two sections, we know that all the possible $\delta$-vectors with $\sum_{i=0}^{d} \delta_{i} \leq 4$ can be obtained by integral simplices, while this does not hold when $\sum_{i=0}^{d} \delta_{i}=5$. Therefore, for the further classifications of the $\delta$-vectors with $\sum_{i=0}^{d} \delta_{i} \geq 5$, it is natural to investigate the $\delta$-vectors of integral simplices. In this 
section, we establish the new equalities and inequalities on $\delta$-vectors for integral simplices when $\sum_{i=0}^{d} \delta_{i}$ is prime. Moreover, by using them, we classify all the possible $\delta$-vectors of integral simplices with $\sum_{i=0}^{d} \delta_{i}=5$ and 7.

The following equalities or inequalities are new constraints on the $\delta$-vectors of integral simplices when $\sum_{i=0}^{d} \delta_{i}$ is prime.

Theorem 5.1 Let $\mathcal{P} \subset \mathbb{R}^{N}$ be an integral simplex of dimension $d$ and $\delta(\mathcal{P})=\left(\delta_{0}, \delta_{1}, \ldots, \delta_{d}\right)$ its $\delta$ vector. Suppose that $\sum_{i=0}^{d} \delta_{i}=p$ is an odd prime number. Let $i_{1}, \ldots, i_{p-1}$ be the positive integers such that $\sum_{i=0}^{d} \delta_{i} t^{i}=1+t^{i_{1}}+\cdots+t^{i_{p-1}}$ with $1 \leq i_{1} \leq \cdots \leq i_{p-1} \leq d$. Then

(a)

$$
i_{1}+i_{p-1}=i_{2}+i_{p-2}=\cdots=i_{(p-1) / 2}+i_{(p+1) / 2} \leq d+1
$$

$$
i_{k}+i_{\ell} \geq i_{k+\ell} \text { for } 1 \leq k \leq \ell \leq p-1 \text { with } k+\ell \leq p-1 .
$$

Example 5.2 When $\sum_{i=0}^{d} \delta_{i}$ is not prime, Theorem 5.1 is not true. In fact, by virtue of Theorem 4.1 $(1,1,0,2,0,0)$ is the $\delta$-vector of some integral simplex of dimension 5 . However, one has $2=i_{1}+i_{1}<$ $i_{2}=3$.

A proof of this theorem is given by considering the additive group $S$ (appeared in Section 2) associated with an integral simplex with prime normalized volume. Since the order of $S$ is equal to the normalized volume of $\mathcal{P}, S$ is nothing but a cyclic group $\mathbb{Z} / p \mathbb{Z}$. By studying $S$ and the degrees of its elements, we obtain the statements (a) and (b). Note that (b) follows from [6, Theorem 2.2], known as CauchyDavenport theorem.

As an application of Theorem 5.1, we give a complete characterization of the possible $\delta$-vectors of integral simplices when $\sum_{i=0}^{d} \delta_{i}=5$ and 7 .

Corollary 5.3 Given a finite sequence $\left(\delta_{0}, \delta_{1}, \ldots, \delta_{d}\right)$ of nonnegative integers, where $\delta_{0}=1$, which satisfies $\sum_{i=0}^{d} \delta_{i}=5$, there exists an integral simplex $\mathcal{P} \subset \mathbb{R}^{d}$ of dimension $d$ whose $\delta$-vector coincides with $\left(\delta_{0}, \delta_{1}, \ldots, \delta_{d}\right)$ if and only if $i_{1}, \ldots, i_{4}$ satisfy

$$
i_{1}+i_{4}=i_{2}+i_{3} \leq d+1,2 i_{1} \geq i_{2} \text { and } i_{1}+i_{2} \geq i_{3},
$$

where $i_{1}, \ldots, i_{4}$ are the positive integers such that $\sum_{i=0}^{d} \delta_{i} t^{i}=1+t^{i_{1}}+\cdots+t^{i_{4}}$ with $1 \leq i_{1} \leq \cdots \leq$ $i_{4} \leq d$.

Corollary 5.4 Given a finite sequence $\left(\delta_{0}, \delta_{1}, \ldots, \delta_{d}\right)$ of nonnegative integers, where $\delta_{0}=1$, which satisfies $\sum_{i=0}^{d} \delta_{i}=7$, there exists an integral simplex $\mathcal{P} \subset \mathbb{R}^{d}$ of dimension $d$ whose $\delta$-vector coincides with $\left(\delta_{0}, \delta_{1}, \ldots, \delta_{d}\right)$ if and only if $i_{1}, \ldots, i_{6}$ satisfy

$$
i_{1}+i_{6}=i_{2}+i_{5}=i_{3}+i_{4} \leq d+1, i_{1}+i_{\ell} \geq i_{\ell+1} \text { for } 1 \leq \ell \leq 3 \text { and } 2 i_{2} \geq i_{4},
$$

where $i_{1}, \ldots, i_{6}$ are the positive integers such that $\sum_{i=0}^{d} \delta_{i} t^{i}=1+t^{i_{1}}+\cdots+t^{i_{6}}$ with $1 \leq i_{1} \leq \cdots \leq$ $i_{6} \leq d$.

By virtue of Theorem 5.1, the "Only if" parts of Corollary 5.3 and Corollary 5.4 are obvious. 


\section{References}

[1] M. Beck and S. Robins, "Computing the Continuous Discretely," Undergraduate Texts in Mathematics, Springer, 2007.

[2] E. Ehrhart, "Polynômes Arithmétiques et Méthode des Polyèdres en Combinatoire," Birkhäuser, Boston/Basel/Stuttgart, 1977.

[3] T. Hibi, "Algebraic Combinatorics on Convex Polytopes," Carslaw Publications, Glebe NSW, Australia, 1992.

[4] T. Hibi, A lower bound theorem for Ehrhart polynomials of convex polytopes, Adv. in Math. 105 (1994), $162-165$.

[5] A. Higashitani, Shifted symmetric $\delta$-vectors of convex polytopes, Discrete Math. 310 (2010), 29252934.

[6] M. B. Nathanson, "Additive number theory, inverse problems and the geometry of subsets," Springer-Verlag, 1996.

[7] A. Schrijver, "Theory of Linear and Integer Programming," John Wiley \& Sons, 1986.

[8] P. R. Scott, On convex lattice polygons, Bull. Austral. Math. Soc. 15 (1976), 395 - 399.

[9] R. P. Stanley, Decompositions of rational convex polytopes, Annals of Discrete Math 6 (1980), 333 -342 .

[10] R. P. Stanley, “Enumerative Combinatorics, Volume 1,” Wadsworth \& Brooks/Cole, Monterey, Calif., 1986.

[11] R. P. Stanley, On the Hilbert function of a graded Cohen-Macaulay domain, J. Pure and Appl. Algebra 73 (1991), $307-314$. 